\title{
Laser looks under the surface of art
}

\section{Technique takes three-dimensional profiles of paintings without taking samples.}

Philip Ball

20 January 2014

Chemists have unveiled a technique that can get under the skin of paintings to provide a three-dimensional analysis of the old masters' works without causing any damage.

Chemist Warren Warren of Duke University in Durham, North Carolina, and his collaborators have adapted an optical-microscopy technique used in medicine for imaging cross-sections of tissue to reveal the structure of paint layers, disclosing which pigments were used and where they sit.

It is a type of spectroscopy in which pulses of visible or near-infrared light — lasting less than a trillionth of a second - pump electrons in some of the atoms or molecules of a sample into higher-energy states. Then a second series of laser pulses probes the way the electrons gradually return to their lower-energy states.

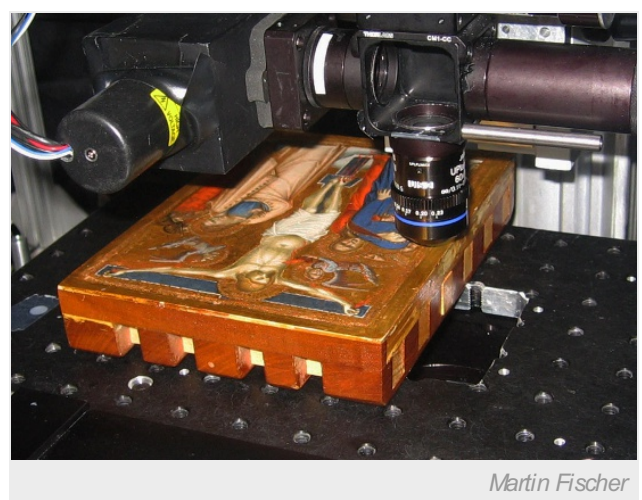

The Crucifixion by fourteen-century artist Puccio Capanna undergoes non-destructive laser scanning that can reveal the structure and composition of different layers of painting.

This 'relaxation' is a complex process that provides a 'fingerprint' of the chemical identity of the region of the region probed - which can be less than half a micrometre wide. In this way the researchers can deduce the composition of individual pigment grains, revealing which materials the artist used. Because the pump and probe pulses can be focused at various depths in the sample, they can produce a chemical profile of paint layers. The results are described in this week's Proceedings of the National Academy of Sciences ${ }^{1}$.

"This method has been in use in chemical-physics laboratories for half a century, but usually required high-power lasers that would be unacceptable for studying art," says Warren. His group has found ways of shaping the laser pulses to make them less intense. Previously its work was geared towards medical applications, but the researchers realized that it could also be used in art conservation. That led Warren to team up with John Delaney, senior imaging scientist at the National Gallery of Art in Washington DC, and William Brown, chief conservator at the North Carolina Museum of Art (NCMA) in Raleigh.

\section{Mixing and layering}

To demonstrate the technique, the researchers first prepared a mock-up of a historical paint layer, a purple made by mixing ultramarine blue (used by medieval and Renaissance painters, when they could afford it) with a modern dye-based red that is chemically similar to a plant dye called madder that artists would have used in the past.
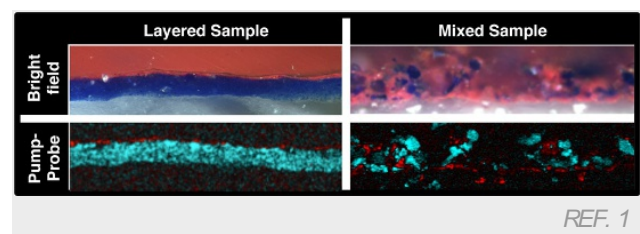

How the new, 'pump-probe' imaging stacks up against traditional, 'bright-field' microscopy: both techniques reveal when paint pigments are layered or mixed (click to enlarge).
The method produced cross-sections that matched those obtained using conventional microscopy methods. It clearly showed the distinction between a purple made by mixing pigments and one in which the red is laid in a thin, translucent glaze over a separate layer of blue - a common technique in medieval and Renaissance art.

The researchers then went on to study a real old painting, the fourteenth-century The Crucifixion by Italian artist Puccio Capanna, which is kept at the NCMA. In an area of the painting depicting a purple robe, the team was able to see layers of ultramarine (under a faded red glaze), iron oxide, ultrathin gold leaf and an iron

mineral used as a 'mordant' to stick the gold leaf to the wooden board. All the findings matched up with a tiny physical cross-section removed to validate the results.

"The current practice is to take as few samples as you can get away with," says Brown. "The ability to take multiple non-destructive 'samples' anywhere on the painting is a tremendous advantage." 
What is more, says Warren, because the method can scan large areas, it can reveal differences in brushstroke thickness, which might distinguish "features put in place by a master artist from those done by students and apprentices".

The laser spectroscopy is not the only non-destructive approach for imaging cross-sections of paintings. X-rays can provide subsurface imaging, for example, but Warren explains that a strong enough beam requires a synchrotron source, of which there are only a few, highly subscribed facilities in the world.

"The preliminary results suggest it is a technique with great potential," says art-conservation scientist Marika Spring of the National Gallery in London. But she cautions that whether it will have important limitations remains to be seen. "It will be interesting to see how generally applicable it proves, once a wider range of paintings have been tested."

Nature | doi:10.1038/nature.2014.14553

\section{References}

1. Villafana, T. E. et al. Proc. Natl Acad. Sci. USA http://dx.doi.org/10.1073/pnas.1317230111 (2014). 